\title{
Algorithm for optimizing the parameters of the maintenance process according to the state with a constant periodicity of control over the criterion of minimum unit cost of operation
}

\author{
GenadiyZhyrov $^{1}$, EvgenyLenkov $^{2}$, Igor Tolok ${ }^{3}$ Ihor Pampukha ${ }^{4}$, HennadiiBanzak $^{5}$, Dmytro Zaitsev ${ }^{6}$ \\ ${ }^{1}$ Department of the Radio Engineering and Radioelectronic Systems of Taras Shevchenko National University of \\ Kyiv, Ukraine, genna-g@ukr.net \\ ${ }^{2}$ Central Scientific Research Institute of the Armed Forces of Ukraine, Ukraine, lenkov85es@ gmail.com \\ ${ }^{3}$ Military Institute of Taras Shevchenko National University of Kyiv, Ukraine, s63010566s@ gmail.com \\ ${ }^{4}$ Military Institute of Taras Shevchenko National University of Kyiv, Ukraine, pamp@ukr.net \\ ${ }^{5}$ Department Metrology and Metrological Support Odessa State Academy of Technical Regulation and Quality, \\ Ukraine, banzakoksana@gmail.com \\ ${ }^{6}$ Military Institute of Taras Shevchenko National University of Kyiv, Ukraine, zaitsev70@ukr.net
}

\begin{abstract}
The problem of extending the service life of complex radio-electronic products and systems remains relevant in our time. Life extension is based on the effective operation of maintenance and repair systems. Each of these systems has its own strategies with corresponding parameters, and these parameters should be different for different products. The criteria that determine the parameters of these systems are often the criteria for minimizing the unit cost of operation and increasing the coefficient of technical utilization. The choice of criterion is determined by the nature of the tasks that the product (system) solves. Also, the parameters of the reliability of the product are significantly influenced by the parameters of the reliability of its component parts, as well as the correct design decisions.
\end{abstract}

The article solves the problem of developing an algorithm for optimizing the parameters of the maintenance process of a radio-electronic product (system) using a maintenance strategy based on a condition with a constant monitoring frequency. The algorithm was developed for the criterion of minimizing the unit operating cost of an object, which is determined for a given period of operation, while ensuring a given requirement for the level of reliability of the object.

Key words: maintenance, reliability, resource extension, maintenance as condition, simulation statistical modeling.

\section{INTRODUCTION}

When designing and developing new products and systems of radio-electronic equipment in the terms of reference, in addition to technical or tactical and technical characteristics, for special-purpose systems, the characteristics of the reliability of the product (system), as well as the cost of operation, are indicated. The main indicators of the reliability of a product are laid down during its design, and the indicators of the reliable operation of such products (systems) are ensured by the operation of the maintenance and repair (MCR) system. Maintenance (MC) and repairs improve reliability and performance, resulting in systematic product life extension. In turn, the cost of operation depends both on the parameters of maintenance, and on the indicators of reliability and maintainability of the product itself. The calculation of indicators of reliability and cost of operation (determination of MC parameters) must be performed at the stage of product design. Thus, there is a problem of optimizing the characteristics of the product (system) and the parameters of its MC, while maintaining the technical characteristics within the established limits [1-4].

In past, a large number of well-known scientists, including Kredentser B.P., BarzilovichE.Yu., Cox D., Smith V., Kashtanov V.O., Ushakov I.O., Barlow R., Proshan F., Strelnikov V.P., Feduhin A.V and others. Recently, many papers in this subject area have also been published. Thus, in [5] presents a new variant of the selective maintenance problem (SMP) to jointly optimize the maintenance planning and resource allocation problems for multiple missions. The classical SMP deals with components and maintenance level selection with the aim of maximizing the system reliability given limited budget and break duration. The resulting SMP addresses five joint decisions: selection of components to maintain, selection of maintenance levels performed on the selected components, identification of breaks where maintenance tasks are performed, repairpersons selection, and maintenance tasks assignment to selected repairpersons. In the [6] present paper, we address these challenges by proposing a conceptual model to enable prescriptive maintenance in semiconductor manufacturing. Different Machine Learning Algorithms are used to predict 
time-to-failure intervals for unplanned downtimes. Furthermore, the concept uses Bayesian Networks to predict the root cause of a failure and ultimately leads to recommendations, which are integrated into maintenance planning routines, in order to increase the system availability by initiating specific maintenance measures. In [7] proposes a condition-based maintenance (CBM) policy with dynamic thresholds and multiple maintenance actions for such a system subject to periodic inspection. The hazard rate is described by the proportional hazards model with a continuous-state covariate process. At each inspection epoch, appropriate action is selected from no maintenance, imperfect maintenance, and preventive replacement based on two dynamic thresholds. Over an inspection interval, the system may experience minor failure or catastrophic failure that can be addressed by minimal repair and corrective replacement, respectively. A modified policy-iteration algorithm is developed to solve the optimization problem in the semi-Markov decision process (SMDP) framework. The effectiveness of the proposed approach is illustrated by a practical numerical example. In [8] presents a sequential imperfect preventive maintenance model to quantify the maintenance efficiency of urban buses. Existing works of sequential imperfect preventive maintenance models are all formulated based on failure intensity reduction. The proposed model in this study defines maintenance efficiency as a proportion of the difference between actual and expected failure intensity increments. Since the proportion of the difference can vary from negative to positive, the proposed model can quantify all kinds of maintenance efficiency given in the existing works. In [9] proposed a maintenance optimization model considering both economic and structural dependencies between components. The impact of structural dependence on the reliability of components was reviewed using a Proportional Hazard Model. The model implemented influencing factors such as component features, the system structure, and the production context. It supported a more accurate reliability assessment. We derived an adaptive maintenance strategy that integrated these dependencies and developed a cost-based optimization model to determine a prudent maintenance plan. We conducted a case study on a spindle of a milling machine to prove feasibility and the model's ability to reliably assess and optimally maintain operations. In [10] developed new approaches to predicting software reliability using the "Knowledge Engineering" approach.

\section{METHODOLOGY}

The task of optimizing the parameters of the maintenance process as it is with a constant frequency of control over the criterion of minimum operating $\operatorname{cost}\left(\min c_{\mathrm{e}}\right)$ mathematically described by the expression (1)

$$
T_{0}\left(E_{\mathrm{mc}}^{*}, U_{\mathrm{mc}}^{*}, T_{\mathrm{c}}^{*}\right) \geq T_{0}^{\mathrm{req}} ; c_{\mathrm{e}}\left(E_{\mathrm{mc}}^{*}, U_{\mathrm{mc}}^{*}, T_{\mathrm{c}}^{*}\right) \rightarrow \min
$$

where: $T_{0}^{\text {req }}$ - requirement for level of reliability objects;
$E_{\mathrm{mc}}^{*}, U_{\mathrm{mc}}^{*}$ and $T_{\mathrm{c}}^{*}$ - desired optimal values of $\mathrm{MC}$ parameters with a constant periodicity of control; $E_{\mathrm{mc}}$ - set elements that are serviced; $U_{\mathrm{mc}}$ - vector of levels that determine the need for maintenance of elements; $T_{\mathrm{c}}$ frequency of control.

Problem (1) is supposed to be solved as a sequence of partial problems in which the set $E_{\mathrm{mc}}$ is fixed. At each step, the auxiliary set $E_{\mathrm{mc}}^{+}$is formed by adding to it one element taken from $E_{\mathrm{mc}}$, and solves the partial problem of determining the optimal parameters that satisfy the condition:

$$
c_{\mathrm{e}}\left(E_{\mathrm{mc}}^{+}, U_{\mathrm{mc}}^{+}, T_{\mathrm{c}}^{+}\right) \rightarrow \min _{U_{\mathrm{mc}}, T_{\mathrm{c}}} .
$$

The solution $\mathrm{P}_{\mathrm{mc}}^{+}=\left\langle E_{\mathrm{mc}}^{+}, U_{\mathrm{mc}}^{+}, T_{\mathrm{c}}^{+}\right\rangle$that satisfies condition (2) is a conditionally optimal solution obtained provided that the set of serviced elements has the composition $E_{\mathrm{mc}}^{+}$, $U_{\mathrm{mc} i}^{+}=\left\{u_{\mathrm{mc1}}^{*}, u_{\mathrm{mc} 2}^{*}, \ldots, u_{\mathrm{mc} i}^{+}\right\}$, where $u_{\mathrm{mc1}}^{*}, u_{\mathrm{mc} 2}^{*}, \ldots, u_{\mathrm{mc} i-1}^{*}$ are the optimal values of the MS levels found in the previous steps of the sequential optimization process, and $u_{\mathrm{mc} i}^{+}$is the conditionally optimal value obtained in the last (i-th step) as a result of solving partial problem (2); $T_{\mathrm{c}}^{+}$- frequency of control, which will satisfy condition (2).

The search for conditionally optimal values $\left\langle u_{\mathrm{mci}}^{+}, T_{\mathrm{c}}^{+}\right\rangle$is carried out in the two-dimensional space $[0,1] \times[0, \infty)$, which significantly simplifies the original problem in its general formulation (1). Since the form of the objective function $c_{\mathrm{e}}$ in

(2) is unknown, we will apply the method of direct search [11-15].

In many cases, partial problem (2) may not have a solution due to the fact that the objective function may not have an extremum on parameter $T_{\mathrm{c}}^{+}$. In addition, due to the fact that the objective function $c_{\mathrm{e}}$ is formed as a result of simulation statistical modeling, its values are subject to random fluctuations, as a result of which the solution of problem (2) is often unstable. The block diagram of the algorithm for solving problem (1) is shown in figure 1.

The source information for the algorithm is a user-specified set of elements $E_{\mathrm{mc}}$ that are subject to or can be serviced, and the required value of the average operating time for failure of the object $T_{0}^{\text {req }}$. 


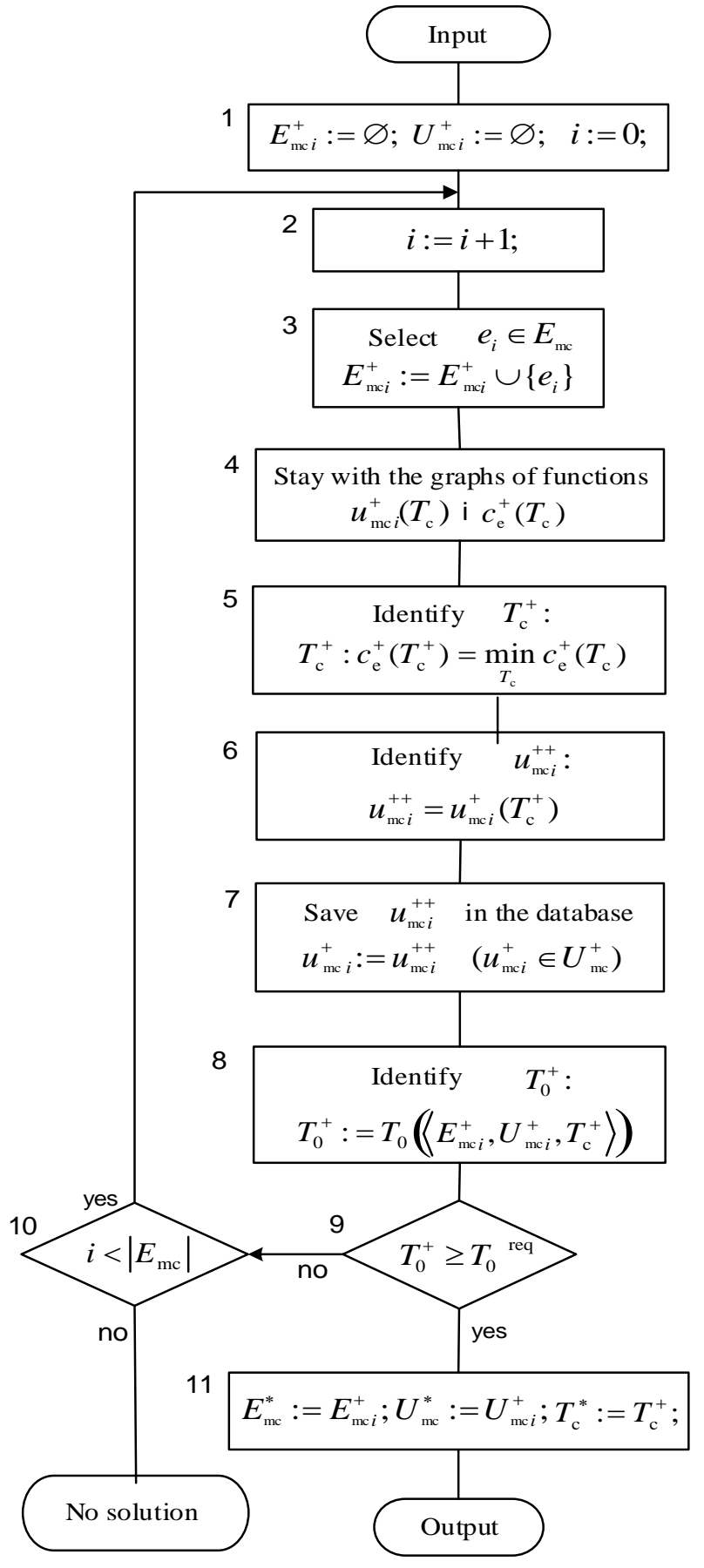

Figure 1: Algorithm for finding the optimal parameters of the maintenance strategy for the state with a constant frequency of control

Operator 1 creates empty sets $E_{\mathrm{mc} i}^{+}$and $U_{\mathrm{mc} i}^{+}$, and initiates the variable $i$, which is used to count the number of steps performed to find a solution (simultaneously, the variable $i$ is the number of the element $U_{\mathrm{mc} i}^{+}$, which is added to the set $E_{\mathrm{mc} i}^{+}$last). The set $E_{\mathrm{mc} i}^{+}$is used as the current set of elements being served (one element from the set $E_{\mathrm{mc}}$ will be added to it at each step). The set $U_{\mathrm{mc} i}^{+}$is used to store the found optimal values of the MC $u_{\mathrm{mc} i}^{+}$levels of the elements included in the set $E_{\mathrm{mc} i}^{+}$.

Operator 2 generates the number $\mathrm{i}$ of the next step of the process of finding the optimal solution.

The operator 3 selects from the set $E_{\mathrm{mc}}$ the $i$-th element $e_{i}$ and adds it to the set $E_{\mathrm{mc} i}^{+}$. The selection of elements $e_{i}$ from the set $E_{\mathrm{mc}}$ is carried out in ascending order of their average operating time before failure.

Operator 4 plots the functions $u_{\mathrm{mc} i}^{+}\left(T_{\mathrm{c}}\right)$ and $c_{\mathrm{e}}^{+}\left(T_{\mathrm{c}}\right)$ depending on the periodicity of MC: $T_{\mathrm{c}}$, where $u_{\mathrm{mc} i}^{+}\left(T_{\mathrm{c}}\right)$ is the optimal value of the MC level for the $i$-th element, found from the condition:

$$
u_{\mathrm{mc} i}^{+}\left(T_{\mathrm{c}}\right): c_{\mathrm{e}}\left(E_{\mathrm{mc} i}^{+}, U_{\mathrm{mc} i}^{+}, T_{\mathrm{c}}\right) \rightarrow \min _{u_{\mathrm{m} c i} \in U_{\mathrm{m} c i}^{+}},
$$

where: $U_{\mathrm{mc} i}^{+}=\left\{u_{\mathrm{mcl}}^{*}, \ldots, u_{\mathrm{mc} i-1}^{*}, u_{\mathrm{mc} i}\right\}$ is a vector in which $u_{\mathrm{mcl}}^{*}, \ldots, u_{\mathrm{mci}-1}^{*}$ are the optimal values of the MC levels of the elements $e_{1}, \ldots, e_{i-1}$ found in the previous steps; $u_{\mathrm{mc} i}$ is a varied MC level for element $e_{i}$, the optimal value of which must be found in accordance with condition (3).

Value $c_{\mathrm{e}}^{+}\left(T_{\mathrm{c}}\right)$ - the specific cost of operation, obtained at the optimal value of the level MC $u_{\mathrm{mc} i}^{+}\left(T_{\mathrm{c}}\right)$ :

$$
c_{\mathrm{e}}^{+}\left(T_{\mathrm{c}}\right)=c_{\mathrm{e}}\left(E_{\mathrm{mci} i}^{+}, U_{\mathrm{mci}}^{+}, T_{\mathrm{c}}\right) .
$$

The vector $U_{\mathrm{mc}}^{+}$in expression (3) differs from $U_{\mathrm{mc} i}^{*}$ only in that in $U_{\mathrm{mc}}^{+}$as the $i$-th component is found the found conditionally optimal value $u_{\mathrm{mc} i}^{+}\left(T_{\mathrm{c}}\right)$.

The operator 5 determines the optimal in the current step value of the periodicity of control $T_{\mathrm{c}}^{+}$, which satisfies the condition:

$$
c_{\mathrm{e}}^{+}\left(T_{\mathrm{c}}^{+}\right)=\min _{T_{\mathrm{c}}} c_{\mathrm{e}}^{+}\left(T_{\mathrm{c}}\right)
$$

Operator 6 determines the optimal value of the MC level $u_{\mathrm{mc} i}^{++}$:

$$
u_{\mathrm{mc} i}^{++}=u_{\mathrm{mc} i}^{+}\left(T_{\mathrm{c}}^{+}\right) .
$$

Operator 7 stores the calculated value $u_{\mathrm{mc} i}^{++}$in the database as the optimal value for this step. 
Operator 8 determines the value reached in the current search step of the average operating time on the failure of the object $T_{0}^{+}: T_{0}^{+}=T_{0}\left(\mathrm{P}_{\operatorname{mcc}(i)}^{+}\right)$.

The operator 9 checks the fulfillment of condition $T_{0}^{+} \geq T_{0}^{\text {req }}$. If this condition is met, then the obtained conditionally optimal solution $\mathrm{P}_{\operatorname{mcc}(i)}^{+}$is taken as the final solution of the problem (operator 11): $\mathrm{P}_{\mathrm{mcc}}^{*}:=\mathrm{P}_{\mathrm{mcc}(i)}^{+}$. The solution process is completed in this case.

Otherwise, if $T_{0}^{+}<T_{0}^{\text {req }}$, the operator 10 is executed, which checks whether all elements of $E_{\mathrm{mc}}$ were used in finding the solution. If all elements have been used, then go to operator 2 and the search continues.

If at some step all the elements selected from $E_{\mathrm{mc}}$ are exhausted ( $i=\left|E_{\mathrm{mc}}\right|$ ), it means that the original problem (1) has no solution, ie the specified requirement $T_{0}^{\text {req }}$ can not be met only by selecting the optimal maintenance parameters for condition.

In order for problem (1) to be solved, it is necessary to: increase the failure rate of the elements of the object; expand the set of elements $E_{\mathrm{mc}}$; or reduce the required failure rate of the object $T_{0}^{\text {req }}$.

\section{RESULT AND DISCUSSION}

The developed algorithm was applied to the test object Test-1, the structure of which is given in [3]. It is assumed that a database has already been created for the object and the necessary initial data have been entered into it. In the object, the set of inhibiting elements $E_{\mathrm{O}}$ includes 15 elements of different levels. For example, the set of potentially serviced elements $E_{\mathrm{mc}}$ includes 5 of the least reliable elements out of $E_{\mathrm{o}}$, the data on which are given in the table 1 . The coefficient of variation of the operating time to failure of all elements is set equal to $v_{i}=0,8$.

Table 1: Characteristics of potentially serviced elements of the Test- 1 object

\begin{tabular}{|c|c|c|c|}
\hline $\mathbf{N}$ & Item name & $\begin{array}{c}\text { Mean time to failure } \\
T_{\text {cp } i}, \mathrm{hr}\end{array}$ & $\begin{array}{c}\text { Item cost } \\
C_{0 i}, \mathrm{c.u} .\end{array}$ \\
\hline 1 & 132 & 10000 & 40 \\
2 & 12 & 14142 & 20 \\
3 & 11111 & 14142 & 20 \\
4 & $131-1$ & 20000 & 10 \\
5 & $131-2$ & 20000 & 10 \\
\hline
\end{tabular}

Other cost and time characteristics for the Test- 1 object are as follows: mean time of replacement of the item of the $i$-th element $\tau_{\text {repi }}=1 \mathrm{hr}$; mean maintenance duration of the $i$-th element $\tau_{m i}=0.2 \mathrm{hr}$; cost of the $i$-element $C_{0 i}=10$ c.u.; cost of replacing the $i$-th element $C_{\text {repli }}=1$ c.u.; cost of the maintenance operation for the $i$-th element $C_{\mathrm{m} i}=1$ c.u.; ( $\forall i=\overline{1,\left|E_{\mathrm{mc}}\right|}$ ); mean time of maintenance control $\tau_{m c}=0.5$ $\mathrm{hr}$; mean time of troubleshooting $\tau_{\text {trou }}=1 \mathrm{hr}$; administrative time for maintenance $\tau_{m a}=1 \mathrm{hr}$. In the absence of MC under the following conditions for the object the following values of indicators are received: $T_{0}=1211 \mathrm{hr} ; c_{\mathrm{e}}=0.03001 \mathrm{c.u} . / \mathrm{hr}$. The required value of the average operating time for failure is equal to $T_{0}^{\text {req }}=1500 \mathrm{hr}$.

According to the developed algorithm, the calculations are performed in the following sequence:

1. In the first step in the set $E_{\mathrm{mc}}^{+}$include one element 132: $E_{\mathrm{mc} 1}^{+}=\{132\}$.

2. The results of testing the algorithm of the 1st step are shown in figure 2.

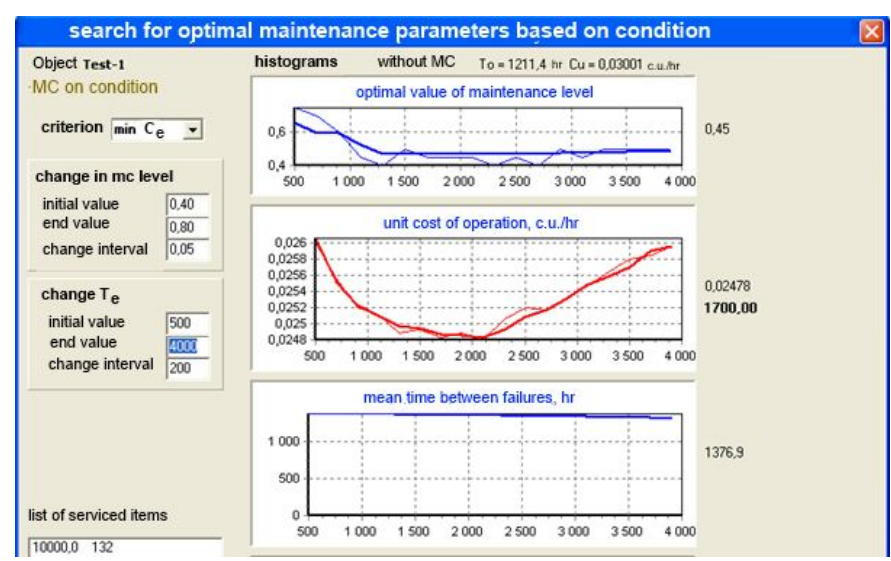

Figure 2: The results of testing the algorithm of the 1st step

According to the graph of function $c_{\mathrm{e}}^{+}\left(T_{\mathrm{c}}\right)$, we determine its minimum value of $c_{\mathrm{e}}^{+}\left(T_{\mathrm{c}}\right)=0.024783 \mathrm{c}$.u. and the corresponding optimal value of $T_{\mathrm{c}}^{+}=1700 \mathrm{hr}$. According to graph $u_{\mathrm{mcl}}^{+}\left(T_{\mathrm{c}}\right)$, we determine the optimal value of $u_{\mathrm{mc} 1}^{++}=u_{\mathrm{mcl}}^{+}\left(T_{\mathrm{c}}^{+}\right)=0.45$. Thus, as a result of performing calculations in the 1st step, we obtain the following conditionally optimal solution:

$$
\mathrm{P}_{\mathrm{mcc}(1)}^{+}=\left\langle E_{\mathrm{mc} 1}^{+}, U_{\mathrm{mc} 1}^{+}, T_{\mathrm{c}}^{+}\right\rangle=\langle\{132\},\{0,45\}, 1700 h r\rangle .
$$

3. According to graph $T_{0}^{+}\left(T_{\mathrm{c}}\right)$, determine the intermediate value of the average operating time to failure 
$T_{0}^{+}=T_{0}\left(\mathrm{P}_{\text {mcc }}^{+}\right)=1376 \mathrm{hr}$.

4. Comparing the obtained value of $T_{0}^{+}=1376 \mathrm{hr}$. with the required value of $T_{0}^{\text {req }}=1500 \mathrm{hr}$, we conclude that the specified requirement is not met. Therefore, it is necessary to continue the calculations and proceed to the next step. We add the following to the set of serviced elements, and adjust the obtained conditionally optimal value $u_{\mathrm{mc} 1}^{++}=0.45$ for the first element.

The results of the algorithm, if the number of serviced elements is equal to five, are given in table 2.

Table 2: The results of calculations of conditionally optimal parameters MCC

\begin{tabular}{|c|c|c|c|c|c|c|}
\hline \multirow{2}{*}{$\begin{array}{c}\text { Step } \\
\text { number } \\
i\end{array}$} & \multicolumn{3}{|c|}{ Conditionally optimal parameters $\mathrm{P}_{\operatorname{mcc}(i)}^{+}$} & \multicolumn{3}{|c|}{$\begin{array}{l}\text { Value of indicators at conventionally optima } \\
\text { parameters } \mathrm{P}_{\operatorname{mcc}(i)}^{+}\end{array}$} \\
\hline & $E_{\mathrm{mc} i}^{+}$ & $u_{\mathrm{mc} i}^{++}$ & $T_{\mathrm{c}}^{+}, \mathrm{hr}$ & $T_{0}^{+}, \mathrm{hr}$ & $c_{\mathrm{e}}^{+}$, c.u. $/ \mathrm{hr}$ & $\varepsilon$ \\
\hline 0 & - & - & - & 1213 & 0,03001 & 0,126 \\
\hline 1 & $\{132\}$ & 0,45 & 1700 & 1376 & 0,02478 & 0,126 \\
\hline 2 & $\{132,12\}$ & $\mathbf{0 , 5 0}$ & 2100 & 1513 & $\mathbf{0 , 0 2 2 0 5}$ & $\mathbf{0 , 1 5 1}$ \\
\hline 3 & $\{132,12,11111\}$ & 0,47 & 1900 & 1693 & 0,01946 & 0,152 \\
\hline 4 & $\{132,12,11111,131-1\}$ & 0,60 & 1900 & 1846 & 0,01803 & 0,157 \\
\hline 5 & $\{132,12,11111,131-1,131-2\}$ & 0,65 & 2100 & 2012 & 0,01665 & 0,163 \\
\hline
\end{tabular}

Taking into account the fact that the requirement for the reliability level $T_{0}^{\text {req }}=1500 \mathrm{hr}$ is set for the object Test-1, according to the obtained data we determine the following optimal maintenance parameters according to criterion $\min c_{\mathrm{e}}: \mathrm{P}_{\text {mcc }}^{*}=\mathrm{P}_{\operatorname{mcc}(2)}^{+}=\langle\{132,12\} ;\{0,45 ; 0,5\}, 2100 h r\rangle$.

At the received optimum parameters of $\mathrm{MCC} \mathrm{P}_{\text {mcc }}^{*}$ the following values of indicators will be provided:

$$
T_{0}\left(\mathrm{P}_{\text {mcc }}^{*}\right)=1513 \mathrm{hr} ; c_{\mathrm{e}}\left(\mathrm{P}_{\text {mcc }}^{*}\right)=0.02209 \text { c.u. } / \mathrm{hr} \text {. }
$$

The statistical error of the simulation results at which these results are obtained is equal to $\varepsilon=0.151$.

\section{CONCLUSION}

The article solves the problem of developing an algorithm for optimizing the parameters of the maintenance process of a radio electronic product (system) using a maintenance strategy for the state with a constant frequency of control. The algorithm is developed for the criterion of minimizing the specific cost of operation of the object, which is determined at a given period of operation, while ensuring a given requirement for the level of failure of the object. The failure rate of an object is determined by the set value of the average failure time. The developed algorithm is based on the application of directed search in the space of maintenance parameters.

\section{REFERENCES}

1. Sergey Lenkov, Igor Tolok, Vadim Tsitsarev, GenadiyZhyrov, EvgenLenkov, YuriiKhlaponin, Bohdan Borowik,"Forecasting reliability of complex technology objects. Parameters optimization of their technical exploitation:" [monograph] in English. Poland, Bielsko-Biala: Publishing house «BEL», 2018. 253 p.
2. Zhyrov G.B. Lenkov E.S., "Algoritmichna model' protsesutehnichnogoobslugovuvanniazastanom $\quad \mathbf{z}$ postiinoiuperiodichnistiukontroliu',Suchasnaspetsial' natehnika: nauk-pr. zhurn. 2017, №1(48), pp. 26-29.

3. GenadiyZhyrov, SerhiiLienkov, YuriiHusak, HennadiiBanzak, Igor Tolok, "Analysis of problem optimization of parameters maintenance process according to state with constant periodicity of control", International Journal of Emerging Trends in Engineering Research. 2020. Vol. 8. №6. - pp. 2606-2611. ISSN: $2347 \quad$ - 3983, https://doi.org/10.30534/ijeter/2020/63862020.

4. Lenkov S., Zhyrov G., Zaitsev D., Tolok I., Lenkov E., Bondarenko T., Gunchenko Y., Zagrebnyuk V., Antonenko O., "Features of modeling failures of recoverable complex technical objects with a hierarchical constructive structure", Eastern-European Journal of Enterprise Technologies. 2017, №4/4 (88), pp. 34-42. DOI: 10.15587/1729-4061.2017.108395

http://journals.uran.ua/eejet/issue/view/6544.

5. K.Chaabane, A.Khatab,C.Diallo, E.-H.Aghezzaf, U.Venkatadri, "Integrated imperfect multimission selective maintenance and repairpersons assignment problem", Reliability Engineering \& System Safety, Volume 199, July 2020, 106895. https://doi.org/10.1016/j.ress.2020.106895.

6. Fabian Biebl, Robert Glawar, AnahidJalali, Fazel Ansari, Bernhard Haslhofer, Peter deBoer, WilfriedSihn, "A conceptual model to enable prescriptive maintenance for etching equipment in semiconductor manufacturing', Procedia CIRP, Volume 88, 2020, pp. 64-69. https://doi.org/10.1016/j.procir.2020.05.012.

7. Rui Zheng, BingkunChen, LiudongGu, "Condition-based maintenance with dynamic thresholds for a system using the proportional hazards model", Reliability Engineering \& System 
Safety, Volume 204, December 2020, 107123. https://doi.org/10.1016/j.ress.2020.107123.

8. Yu Zhou, Gang Kou, Hui Xiao, Yi Peng, FawazE.Alsaadi, "Sequential imperfect preventive maintenance model with failure intensity reduction with an application to urban buses", Reliability Engineering \& System Safety, Volume 198, June 2020, 106871. https://doi.org/10.1016/j.ress.2020.106871.

9. Duc-HanhDinh, PhucDo, BenoitIung, "Maintenance optimisation for multi-component system with structural dependence: Application to machine tool sub-system", CIRP Annals, 2020, Article in pres. https://doi.org/10.1016/j.cirp.2020.04.004.

10. MayuriH.Molawade, Dr. Shashank D. Joshi, "Software reliability prediction using Knowledge Engineering approach",International Journal of Advanced Trends in Computer Science and Engineering, 2019, Volume 8, No.6, pp. 2768-2772. https://doi.org/10.30534/ijatcse/2019/14862019.

11. . Bandi B, "Metodyoptimizatsii. Vvodnyikurs", M.: Radio isviaz', 1988, 128 p.

12. Shup T, "Reshenieinzhenernyhzadachna EVM: prakticheskoerukovodstvo", M.: Mir, 1982, 238 p.

13. Bohdan Zhurakovskyi, JuliyBoiko, VladymirDruzhynin, Irina Zeniv, OleksanderEromenko, "Increasing the efficiency of information transmission in communication channels", Indonesian Journal of Electrical Engineering and Computer Science, vol.19, no 3, p.1306-1315, Sept. 2020. 10.1109/IEEECONF48371.2020.9078564.

14. Lienkov S. V., Zhirov H. B., Tolok I. V., Lienkov Ye. S., "Simulation model of the adaptive maintenance procedure of complex radioelectronic facilities", Radioelektronika, informatika, upravlenie. 2020, №1(48), pp. 63-75. DOI 10.15588/1607-3274-2020-1-7. http://ric.zntu.edu.ua/article/view/201600.

15. Elvin Yano, Louise Sebastian Reyes, Paul Randell Castro,Joseph Joshua Tarroza, Anthony James Bautista, "Prototype of a Compact Assistant Surveillance Robot for Search and Rescue Operations", International Journal of Advanced Trends in Computer Science and Engineering, 2020, Vol. 9, no.1.1, pp.64-68. https://doi.org/10.30534/ijatcse/2020/1291.12020. 